\title{
Evaluating the effect of immune cells on the outcome of patients with mesothelioma
}

\begin{abstract}
Serena J Chee ${ }^{1,2}$, Maria Lopez ${ }^{1,3}$, Toby Mellows ${ }^{3,4}$, Sharmali Gankande ${ }^{3}$, Karwan A Moutasim ${ }^{1,3}$, Scott Harris ${ }^{5}$, James Clarke ${ }^{1}$, Pandurangan Vijayanand ${ }^{4}$, Gareth J Thomas ${ }^{1,6,7}$ and Christian H Ottensmeier ${ }^{*} 1,2,6,7$

${ }^{1}$ Cancer Sciences Unit, Faculty of Medicine, University of Southampton, Tremona Road, Southampton SO16 6YD, UK; ${ }^{2} \mathrm{NIHR}$ Southampton Biomedical Research Centre, Tremona Road Southampton General Hospital Southampton SO16 6YD, UK; ${ }^{3}$ Department of Cellular Pathology, University Hospital Southampton NHS Foundation Trust, Tremona Road, Southampton SO16 6YD, UK; ${ }^{4}$ Clinical and Experimental Sciences, Southampton National Institute for Health Research Respiratory Biomedical Research Unit, University of Southampton, Faculty of Medicine, Southampton SO16 6YD, UK; ${ }^{5}$ Public Health Sciences and Medical Statistics, University of Southampton, Tremona Road, Southampton SO16 6YD, UK and ${ }^{6}$ NIHR CRUK Experimental Cancer Medicine Centre Southampton, Tremona Road, Southampton SO16 7YD, UK
\end{abstract}

Background: We systematically assessed the prognostic and predictive value of infiltrating adaptive and innate immune cells in a large cohort of patients with advanced mesothelioma.

Methods: A tissue microarray from 302 samples was constructed. Markers of adaptive immune response in T-cells (CD8 ${ }^{+}$, $\left.\mathrm{FOXP3}^{+}, \mathrm{CD}^{+}, \mathrm{CD} 4 \mathrm{RO}^{+}, \mathrm{CD}^{+}\right)$and $\mathrm{B}$-cells $\left(\mathrm{CD} 2 \mathrm{O}^{+}\right)$, and of innate immune response; neutrophils $\left(\mathrm{NP} 57^{+}\right)$, natural killer cells $\left(\mathrm{CD} 56^{+}\right)$and macrophages $\left(\mathrm{CD} 68^{+}\right)$were evaluated.

Results: We found that in the epithelioid tumours, high $\mathrm{CD} 4^{+}$and $\mathrm{CD} 20^{+}$counts, and low FOXP3 ${ }^{+}, \mathrm{CD} 68^{+}$and $\mathrm{NP} 57^{+}$counts linked to better outcome. In the non-epithelioid group low $\mathrm{CD}^{+}$and low FOXP3 ${ }^{+}$counts were beneficial. On multivariate analysis low FOXP3 ${ }^{+}$remained independently associated with survival in both groups. In the epithelioid group additionally high $\mathrm{CD}^{+}$, high $\mathrm{CD}_{2} \mathrm{O}^{+}$, and low $\mathrm{NP} 57^{+}$counts were prognostic.

Conclusions: Our data demonstrate for the first time, in predominately advanced disease, the association of key markers of adaptive and innate immunity with survival and the differential effect of histology. A better understanding of the immunological drivers of the different subtypes of mesothelioma will assist prognostication and disease-specific clinical decisionmaking.

Mesothelioma is a malignancy most commonly affecting the pleura, but can also arise in the peritoneum, tunica vaginalis and pericardium. It is mainly associated with asbestos exposure with a lag time from exposure to diagnosis of up to 30-40 years (Scherpereel et al, 2010). There is also a genetic component, with family clusters of patients who possess BAP1 mutations having an increased likelihood of developing mesothelioma and uveal melanoma (Testa et al, 2011).

Mesothelioma incidence is predicted to peak in the United Kingdom in 2020. Worldwide however, the incidence will continue to rise as asbestos is still mined and used in industry in many countries outside of Europe, Australia and the United States. It is not yet clear if inhalation of carbon nanotubes, increasingly used in the fields of electronics and medicine, cause similar sequalae as inhalation of asbestos fibres (Jaurand et al, 2009).

Mesothelioma usually presents late, with diagnosis typically made by a combination of radiological findings and pleural biopsy/ pleural fluid cytology. The median survival following a diagnosis of mesothelioma is $4-18$ months despite treatments like chemotherapy, radiotherapy and surgery. 
Asbestos-associated pleural mesothelioma results from chronic inflammation, due to the body's inability to deal with a foreign antigen in the form of inhaled asbestos fibres. Three histological subtypes of mesothelioma are recognised. Epithelioid mesothelioma carries the best prognosis, sarcomatoid mesothelioma is the most aggressive and tumours with mixed morphology have intermediate outcomes.

The role of the immune system in cancer is well established and is critically involved in immunoediting and surveillance; tumour progression results from immune escape (Dunn et al, 2004; Schreiber et al, 2011). In many solid tumours, including colorectal, lung, breast and oropharyngeal, tumour infiltrating lymphocytes (TILs) confer a survival benefit (Galon et al, 2006; Al Shibli et al, 2008; Gooden et al, 2011; García-Martínez et al, 2014; Ward et al, 2014). A meta-analysis of 23 studies of solid tumours demonstrated the presence of $\mathrm{CD}^{+}$TILs conferred a prognostic advantage and a similar analysis of six studies of CD4 ${ }^{+}$TILs showed a significant effect on overall survival (Gooden et al, 2011). A more recent meta-analysis of nearly 18000 tumours in 39 cancers found higher levels of $\mathrm{T}$-cell fractions to be generally associated with better survival (Gentles et al, 2015).

In mesothelioma, the impact of TIL density is less clear. Previous small surgical studies have suggested that tumour CD8 ${ }^{+}$ T-cell infiltration is associated with better survival (Anraku et al, 2008; Yamada et al, 2010). More recently, a larger series demonstrated tumour $\mathrm{CD}^{+}{ }^{+} \mathrm{T}$-cell infiltration confers a survival advantage in epithelioid mesothelioma (Uijie et al, 2015). These three cohorts consisted mainly of patients who were fit enough to undergo surgical resection.

The link between T-cell density in the tumour and outcome for advanced disease is not resolved. In the study presented here we sought to characterise the link between morphological density of immune cells in mesothelioma and outcome in a predominately treatment naïve cohort. We undertook an assessment of innate and adaptive immune cells in a cohort of 302 patients from a large UK centre, using immunohistochemical evaluation of immune cells.

Establishing which immune cells are associated with clinical outcome may also have a bearing on the evaluation of response to treatments such as chemotherapy and immunotherapy. Immunotherapy trials in mesothelioma are underway with checkpoint inhibitors such as tremelimumab targeting CTLA4 or pembrolizumab directed against PD-1 (Calabrò et al, 2013; Alley et al, 2017). Similarly, targeting WT1 (Zauderer, 2010) and mesothelin (Kelly et al, 2012; Hassan et al, 2015) by vaccination or CAR (Rosenberg, 2012) therapies is an area of intense interest. To date, the factors that are associated with treatment success are not yet fully understood. Where data regarding chemotherapy was available, we evaluated whether particular immune cells were associated with treatment success.

\section{MATERIALS AND METHODS}

Case selection. Ethical approval for this study (NRES Southampton and South West Hampshire LREC 10/H0504/32) was in place at our institution.

Patients included in the cohort had a pathological diagnosis of mesothelioma dated at least 2 years prior to analysis. A total of 302 consecutive formalin fixed paraffin embedded tissue blocks archived between 2004 and 2012 were assessed by haematoxylineosin staining to ensure they contained sufficient tissue for further immunohistochemical evaluation. A tissue microarray (TMA) was generated from this cohort. Some cases could not be evaluated as there was tissue loss in the formation of the TMA. Cases were included in the analysis if at least three high-powered fields were available. A subcohort of $\sim 170$ cases was evaluated for $\mathrm{CD}^{+}$
T-cells and $\mathrm{CD}^{+}{ }^{+}$natural killer cells. All samples were taken at the time of diagnostic biopsy or surgical intervention when no systemic treatment had been given.

Clinical data were collected from patient records. Where survival data were not available locally, the date and cause of death was retrieved from the national cancer registry (Public Health England) following a formal data-access application.

Post diagnosis treatment data were available from 166 patients. Of these $61(37 \%)$ underwent chemotherapy and 105 (63\%) did not. Treatment data were not available for 136 patients who had been referred to our centre for diagnostic procedures but were then managed at other sites (Table 1 ).

Of the 302 patients, 259 (86\%) underwent palliative symptom management with thoracoscopy and pleural biopsy followed by talc pleurodesis; and 7 (2\%) underwent image guided pleural biopsy. Thirty-six (12\%) underwent a radical surgical approach including decortication/pleurectomy/pleuro-pneumonectomy (Table 1).

Patients were excluded from the analysis based on the following criteria: tissue loss resulting in availability of less than three highpowered fields, unavailability of survival data, death within 30 days of biopsy, lack of treatment data in the post diagnosis treatment group and an undefined histological morphology.

Histopathological analysis-tissue microarray. Tumour histology was re-reviewed by pathologists GJT, KAM and SG. Haematoxylineosin stained slides from all available cases were assessed and three representative areas of tumour selected.

Triplicate $1 \mathrm{~mm}$ cores were taken from the corresponding formalin fixed paraffin embedded tissue block using a semiautomated system (Aphelys Minicore 2, Mitogen, Harpenden, UK) to generate a TMA. TMA sections $(4 \mu \mathrm{m})$ were used for haematoxylin-eosin and immunohistochemical staining.

\begin{tabular}{|c|c|c|c|c|}
\hline Variable & $N(\%)$ & $\begin{array}{c}\text { Median OS } \\
\text { days } \\
\text { (months) }\end{array}$ & $95 \% \mathrm{Cl}$ & $P$-value \\
\hline Overall group & $302(100 \%)$ & $278(9.2)$ & $247-308$ & \\
\hline $\begin{array}{l}\text { Age } \\
\qquad<65 \\
\geqslant 65\end{array}$ & $\begin{array}{l}63(21 \%) \\
239(79 \%)\end{array}$ & $\begin{array}{l}349(11.6) \\
263(8.7)\end{array}$ & $\begin{array}{l}257-440 \\
229-296\end{array}$ & 0.157 \\
\hline $\begin{array}{l}\text { Sex } \\
\quad \text { Male } \\
\text { Female }\end{array}$ & $\begin{array}{l}243(81 \%) \\
59(19 \%)\end{array}$ & $\begin{array}{l}275(9.2) \\
291(9.7)\end{array}$ & $\begin{array}{l}243-306 \\
194-387\end{array}$ & 0.682 \\
\hline $\begin{array}{l}\text { Subtype } \\
\text { Epithelioid } \\
\text { Non-epithelioid } \\
\text { Biphasic } \\
\text { Sarcomatoid } \\
\text { Undefined }\end{array}$ & $\begin{array}{l}172(57 \%) \\
130(43 \%) \\
82(27 \%) \\
41(14 \%) \\
7(2 \%)\end{array}$ & $\begin{array}{l}342(11.4) \\
205(6.8) \\
216(7.2) \\
187(6.2)\end{array}$ & $\begin{array}{l}277-406 \\
146-260 \\
152 \text { to } 279 \\
112 \text { to } 261\end{array}$ & $<0.0001$ \\
\hline \multicolumn{5}{|l|}{ Intervention } \\
\hline $\begin{array}{l}\text { Chemotherapy } \\
\text { status known } \\
\text { Overall } \\
\text { Yes } \\
\text { No }\end{array}$ & $\begin{array}{l}166 \\
61(37 \%) \\
105(63 \%)\end{array}$ & $\begin{array}{l}280(9.3) \\
460(15.3) \\
205(6.8)\end{array}$ & $\begin{array}{l}239-320 \\
360-559 \\
159-250\end{array}$ & $<0.0001$ \\
\hline $\begin{array}{l}\text { Chemotherapy } \\
\text { status unknown }\end{array}$ & & 136 & & \\
\hline $\begin{array}{l}\text { Surgery } \\
\text { Overall } \\
\text { Radical } \\
\text { Palliative }\end{array}$ & $\begin{array}{l}295 \\
36(12 \%) \\
259(86 \%)\end{array}$ & $\begin{array}{l}279(9.3) \\
330(11.0) \\
274(9.1)\end{array}$ & $\begin{array}{l}247-310 \\
185-474 \\
242-305\end{array}$ & 0.735 \\
\hline No surgery & & $7(2 \%)$ & & \\
\hline
\end{tabular}


Immunohistochemistry was performed using an automated platform (Dako Autostainer) in a CPA-accredited clinical cellular pathology department using antibodies optimised to national diagnostic standards (NEQAS). The antibodies used were antihuman CD3 1:200 (clone F7.2.38; Dako, Carpinteria, CA, USA), anti-human CD4 1:50 (clone 4B, Dako), CD8 RTU (Clone C8/14 4B, Dako), CD20 1:250 (Clone L26, Dako), CD45RO 1:2500 (Clone UCHL-1, Dako), CD56 RTU (Clone 123C3, Dako), NP57 1:100 (Dako), CD68 RTU (Clone PG-M1, Dako), Wilms Tumour 1 RTU (Clone 6F-H2, Dako), FoxP3 1:100 (Clone 236A/E7, Abcam, Cambridge, UK).

Triplicate random high-power fields $(\times 400)$ were counted manually per core across three cores on the Olympus dotSlide (SC). The average of 3-9 counts per patient was calculated to allow for intra-tumoural heterogeneity. The variability in the number of counts per patient relates to the number of viable cores present on the TMA.

The mean of each antibody count was taken as the cutoff point between high and low counts. Representative high and low T-cell densities are shown in Figure 1.

Statistical analysis. Statistical analyses were performed using SPSS Version 22 (IBM Corp. Released 2013. IBM SPSS Statistics for Windows, Version 22.0, IBM Corp., Armonk, NY, USA). Survival time was measured from the time of diagnosis to the time of death. Patients for whom the date of death was unknown were censored from the survival analyses. Kaplan-Meier plots with log-rank tests, univariate and multivariate Cox proportional hazard models were used to analyse the survival data. Separate subtype models were fitted due to the presence of interactions between subtype and several of the immune markers analysed. A $P$-value of $<0.05$ was considered significant.

Our cohort was divided into epithelioid and non-epithelioid (biphasic and sarcomatoid) mesothelioma subtypes for the purposes of analysis as there were only 41 cases of sarcomatoid mesothelioma.

\section{RESULTS}

Clinical and demographic characteristics of our patient cohort are shown in Table 1 . The median age was 72 (41-90) years, $81 \%$ of the patients were men. Of the 302 tumours, 172 were classified as epithelioid (57\%), 82 as biphasic (27\%) and 41 as sarcomatoid (14\%) subtypes. Seven cases (2\%) had an undefined morphology. The median follow-up was 278 days (9.3 months) and the minimum follow-up time was 30 days. There were 293 deaths from

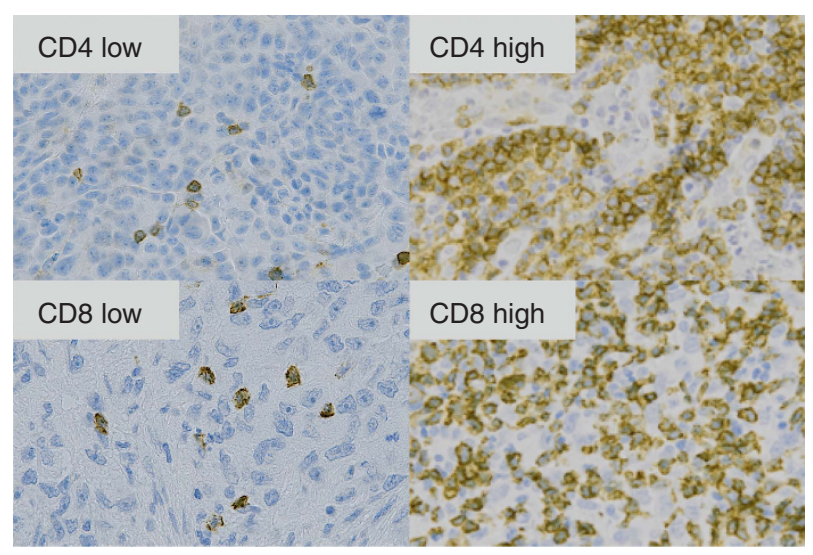

Figure 1. Representative low and high T-cell densities $(\times 400)$ magnification: $\mathrm{CD} 4^{+}$and $\mathrm{CD} 8^{+}$cells. mesothelioma over the study period. Survival analysis by histological subtype was consistent with published data. The median overall survival for epithelioid mesothelioma in our cohort was 342 days (11.4 months) (95\% CI 277-406 days) and for nonepithelioid mesothelioma was 205 days (6.8 months) (95\% CI 146260 days) (Table 1$)$.

The adaptive immune response, particularly involving cytotoxic T-cells, has been shown to influence the prognosis of many tumour types. We examined expression of markers for cytotoxic T-cells $\left(\mathrm{CD} 8^{+}\right)$, regulatory $\mathrm{T}$-cells $\left(\mathrm{FOXP} 3^{+}\right)$, helper $\mathrm{T}$-cells $\left(\mathrm{CD} 4^{+}\right)$, memory T-cells $\left(\mathrm{CD}_{5} \mathrm{RO}^{+}\right)$, T-cells $\left(\mathrm{CD}^{+}\right)$and B-cells $\left(\mathrm{CD} 20^{+}\right)$in the epithelioid and non-epithelioid subtypes.

The mean count/high-power field was used to define the cut point between high and low values per marker evaluated. Median overall survival with $95 \%$ confidence interval and $P$-values for the epithelioid and non-epithelioid groups are shown in Table 2.

In the epithelioid group, we found that high $\mathrm{CD}^{+}(P=0.005)$, low $\mathrm{FOXP}^{+}(P=0.024)$ and high ${ }^{+} \mathrm{CD} 20^{+}$counts $(P=0.008)$ were associated with a better outcome. The relevant Kaplan-Meier curves are shown in Figure 2A-C. These markers were associated with a longer overall survival of 7, 3 and 7 months respectively compared to the $\mathrm{CD}^{+}$low, $\mathrm{FOXP}^{+}$high and $\mathrm{CD} 20^{+}$low groups. $\mathrm{CD}^{+}{ }^{+} \mathrm{T}$-cell counts were not associated with survival in the epithelioid group $(P=0.983)$.

In the non-epithelioid group, positive prognostic markers were low $\mathrm{CD}^{+} \quad(P=0.019)$ and low FOXP3 ${ }^{+}(P=0.012)$ T-cell counts. Kaplan-Meier curves are shown in Figure 3A and B. Both markers were associated with a 4-month longer overall survival in the non-epithelioid group compared to the $\mathrm{CD}^{+}$high and FOXP3 ${ }^{+}$high groups.

The tumour microenvironment also contains innate immune cells and we evaluated the expression of markers of neutrophils $\left(\mathrm{NP}_{5}{ }^{+}\right)$, natural killer cells $\left(\mathrm{CD}^{+} 6^{+}\right)$and macrophages $\left(\mathrm{CD}^{+}\right)$.

In the epithelioid group, we found the positive predictors of outcome from innate immune cells were low $\mathrm{CD}^{+} 8^{+}(P=0.026)$ and low $\mathrm{NP}^{+}(P=0.006)$ counts. Kaplan-Meier curves are shown in Supplementary Figure 1A and B. These markers were both associated with a 3-month longer overall survival in the epithelioid group compared to the $\mathrm{CD}^{+} 8^{+}$high and $\mathrm{NP}^{+} 7^{+}$high groups. In the non-epithelioid group, none of the markers of innate immunity tested were significantly associated with survival.

Wilms tumour 1 (WT1) is expressed in most cases of mesothelioma. Expression was not associated with differential survival in either morphology in our data set.

Multivariate analysis was performed next based on the parameters that were significant on univariate analysis. In the epithelioid group, a high $\mathrm{CD}^{+}(P=0.003)$, high $\mathrm{CD} 20^{+}$ $(P=0.010)$, low $\mathrm{FOXP3}^{+} \quad(P=0.000414)$ and low $\mathrm{NP}^{+} 7^{+}$ $(P=0.038)$ counts remained significantly associated with survival. In the non-epithelioid group, a low number of $\mathrm{FOXP}^{+}$cells associated with survival, $P=0.043$. The data for the multivariate analysis of both groups is shown in Table 3.

The ratio of $\mathrm{CD} 4^{+} / \mathrm{CD}^{+}$with a cut point of 1 between low and high counts was analysed. A ratio of $>1$ was associated with longer survival only in the epithelioid group, 99 low, 59 high (158 total) $P=0.047$. Kaplan-Meier curves are shown in Supplementary Figure $2 \mathrm{~A}$ and $\mathrm{B}$.

Of 166 patients managed in our own centre, 61 had received chemotherapy. These 61 chemotherapy-treated patients had a significantly better survival than those who were not given chemotherapy $(P<0.0001)$, likely reflecting both patient selection and effect of treatment. In the 61 patients treated with chemotherapy, a high $\mathrm{CD}^{+}{ }^{+}$count identified patients who lived longer $(P=0.034)$; no effect was seen for $\mathrm{CD} 8^{+} \mathrm{T}$-cells.

Of 94 patients with epithelioid histology, 42 were treated with chemotherapy. Chemotherapy administration but also high $\mathrm{CD} 4{ }^{+}$, 
Table 2. Univariate analysis of overall survival and immune parameters

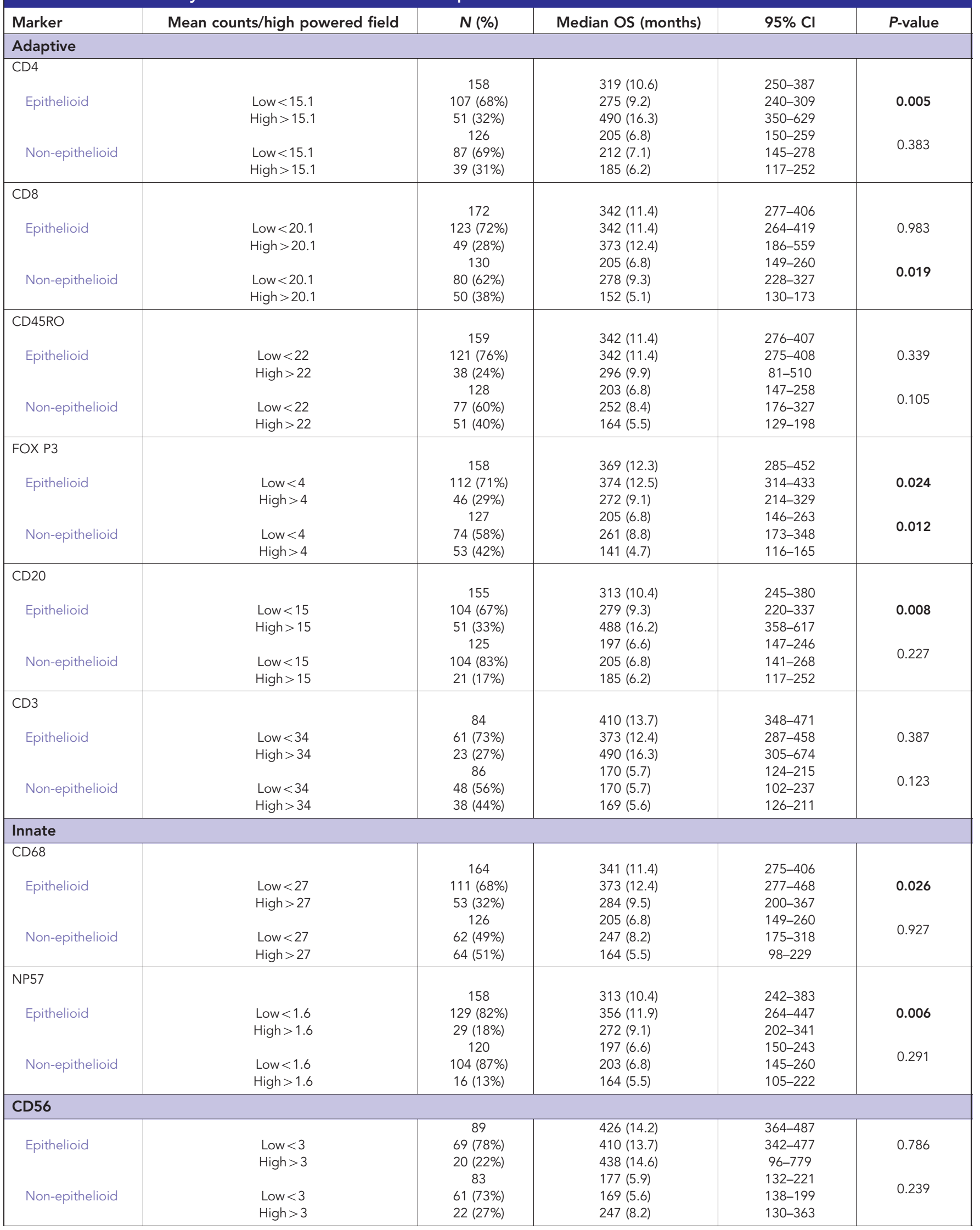




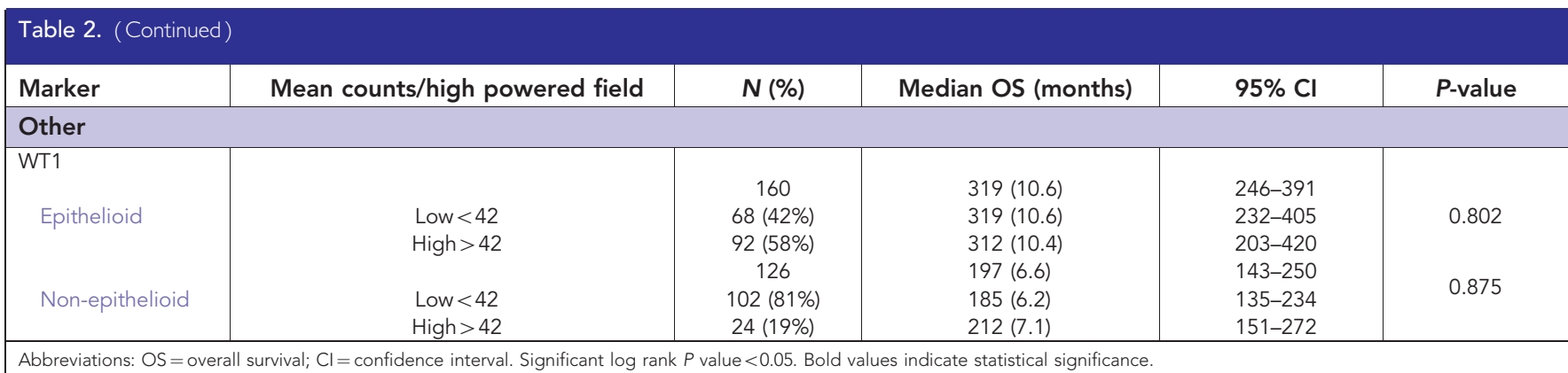
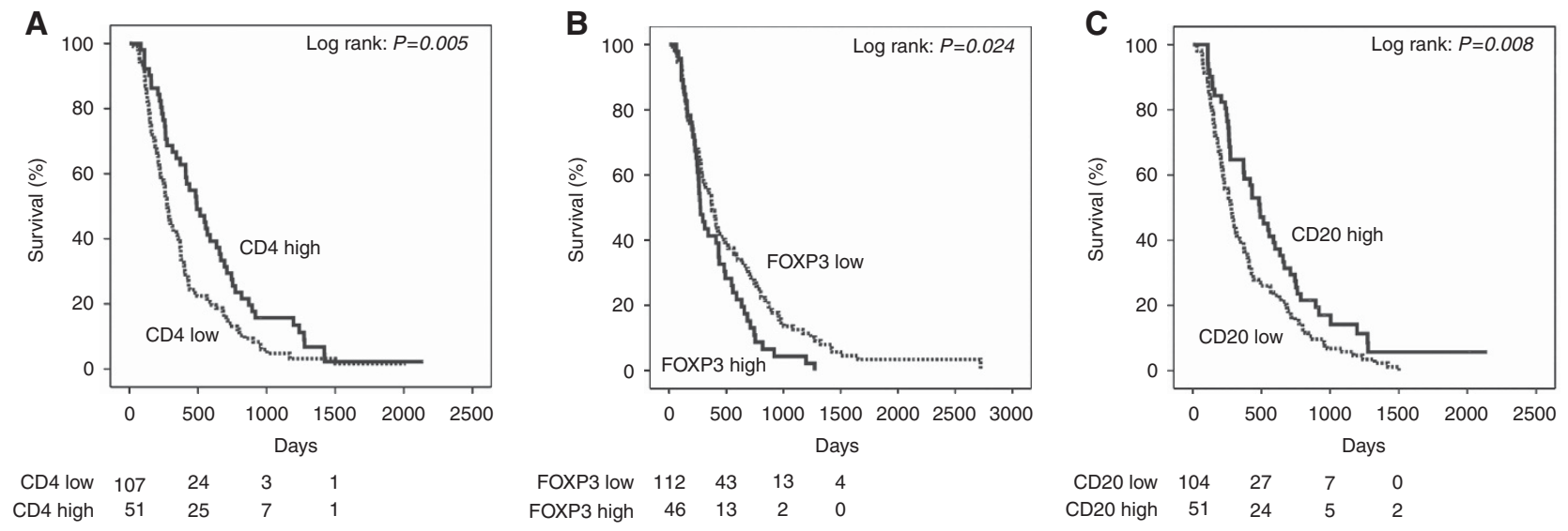

Figure 2. Kaplan-Meier survival curves of adaptive immune markers associated with survival in epithelioid mesothelioma. (A) Kaplan-Meier curves for epithelioid mesothelioma survival according to CD4 ${ }^{+}$T-cell counts (log-rank test, $P=0.005$ ). (B) Kaplan-Meier curves for epithelioid mesothelioma survival according to FOXP3 ${ }^{+}$T-regulatory cell counts (log-rank test, $\left.P=0.024\right)$. (C) Kaplan-Meier curves for epithelioid mesothelioma survival according to $\mathrm{CD}_{20}{ }^{+}$B-cell counts (log-rank test, $P=0.008$ ).
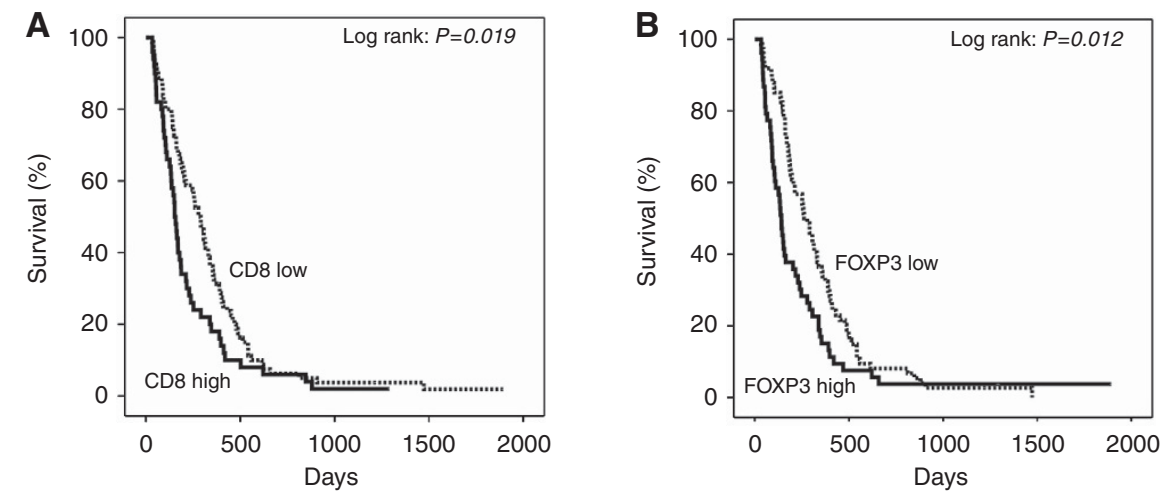

$\begin{array}{rrrrr}\text { CD8 low } & 80 & 13 & 3 & 1 \\ \text { CD8 high } & 50 & 4 & 1 & 0\end{array}$

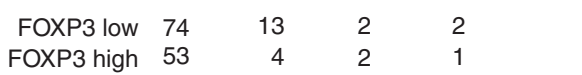

Figure 3. Kaplan-Meier survival curves of adaptive immune markers associated with survival in non-epithelioid mesothelioma. (A) Kaplan-Meier curves for non-epithelioid mesothelioma survival according to $\mathrm{CD}^{+}{ }^{+}$T-cell counts (log-rank test, $\left.P=0.019\right)$. (B) Kaplan-Meier curves for nonepithelioid mesothelioma survival according to FOXP3 ${ }^{+}$T-regulatory cell counts (log-rank test, $P=0.012$ ).

high $\mathrm{CD} 20^{+}$, low $\mathrm{FOXP}^{+}$counts were independent prognostic factors $(P=0.001, P=0.005, P=0.015, P=0.046)$ in multivariate analysis. In the group of 72 non-epithelioid cases, 19 patients had been treated with chemotherapy; only chemotherapy administration was independently associated with survival $(P=0.029)$.

\section{DISCUSSION}

We have evaluated a large unselected cohort of patients for evidence of whether immune attack occurs in mesothelioma and how this might affect survival. To our knowledge, this is the largest cohort of mesothelioma containing all subtypes analysed to date. Our data represent a patient population in whom treatment was given with palliative intent from the outset (88\%). Only $37 \%$ of the cohort received palliative chemotherapy and our cohort is clinically distinct from published data sets that have evaluated TIL density in mesothelioma to date and where the focus has been on operable disease (Anraku et al, 2008; Yamada et al, 2010; Uijie et al, 2015).

We studied markers of adaptive $\left(\mathrm{CD}^{+}, \mathrm{CD}^{+}, \mathrm{CD}^{+}, \mathrm{T}\right.$ regulatory, $\mathrm{T}$ memory and $\mathrm{B}$-cells) and innate immunity (macrophages, natural killer cells and neutrophils). Epithelioid and 
Table 3. Multivariate analysis of overall survival in whole cohort

\begin{tabular}{|l|l|c|c|c|}
\hline & Marker & HR & 95\% Cl & P-value \\
\hline \multirow{5}{*}{ Epithelioid $n=131$} & CD4 & 0.499 & $0.313-0.795$ & 0.003 \\
& FOX P3 & 2.399 & $1.476-3.900$ & $<0.001$ \\
& CD20 & 0.560 & $0.361-0.869$ & 0.010 \\
& CD8 & 0.939 & $0.579-1.522$ & 0.797 \\
& CD68 & 1.325 & $0.909-1.930$ & 0.143 \\
& NP 57 & 1.595 & $1.026-2.481$ & $\mathbf{0 . 0 3 8}$ \\
\hline \multirow{5}{*}{ Non-epithelioid $n=111$} & CD4 & 0.800 & $0.465-1.375$ & 0.420 \\
& FOX P3 & 1.554 & $1.015-2.381$ & 0.043 \\
& CD20 & 1.122 & $0.619-2.034$ & 0.704 \\
& CD8 & 1.470 & $0.939-2.302$ & 0.092 \\
& CD68 & 0.961 & $0.646-1.429$ & 0.843 \\
& NP 57 & 1.338 & $0.769-2.325$ & 0.303 \\
\hline
\end{tabular}

Abbreviations: $\mathrm{HR}=$ hazard Ratio; $\mathrm{Cl}=$ confidence interval. Cox proportional hazards regression model, significant $P$ value $<0.05$. Bold values indicate statistical significance.

non-epithelioid mesothelioma are clinically distinct diseases, and our data demonstrate they are immunologically different also; adaptive immune cell infiltrates differentially link to outcome between subtypes.

A limitation to this study is the analysis is based on staining small biopsy specimens in the TMA which may not be representative of the whole tumour. This is important as mesothelioma has been shown to be polyclonal in origin (Comertpay et al, 2014). We attempted to correct for intertumoural heterogeneity by taking three cores across the tumour and 3-9 counts per patient.

Unexpectedly, high $\mathrm{CD}^{+}$density appears not to be beneficial for survival in mesothelioma patients. In the epithelioid group the $\mathrm{CD}^{+}$T-cell density was indifferent for outcome; in the nonepithelioid group, a low number of $\mathrm{CD}^{+}$T-cells linked with a survival advantage, although this observation was not maintained in the multivariate analysis. This is in contrast to observations in many other solid cancers where a high density of CD8 ${ }^{+}$TILs has been shown to confer a survival advantage (Galon et al, 2006; Al Shibli et al, 2008; Gooden et al, 2011; García-Martínez et al 2014; Ward et al, 2014). Published data suggest that non-epithelioid mesothelioma is more likely to be PD-L1 positive and to have a higher proportion of proliferating $\mathrm{CD}^{+}{ }^{+} \mathrm{T}$-cells (Awad et al, 2016). This suggests that in our data the presence of CD ${ }^{+} \mathrm{T}$-cells is also likely to be associated with high PD-L1 expression. It is unclear whether such PDL1 expression in the tumour cells is in part or even mostly a reflection of immune attack via IFN signalling from immune cells (and therefore reflects immunogenicity of the cancer tissue) or conversely whether PDL1 expression might be driven by another pathway in mesothelioma, with only deleterious effects on T-cell responses.

A high density of CD4 ${ }^{+}$TILs conferred a survival advantage in the epithelioid group, both on univariate and multivariate analysis. $\mathrm{CD}^{+}{ }^{+} \mathrm{T}$-cells are important for activating a range of tumourreactive immune cells including $\mathrm{CD}^{+}{ }^{+} \mathrm{T}$-cells and $\mathrm{B}$-cells (Ding et al, 2010). Further work to establish the targets of CD4 ${ }^{+}$T-cell recognition is needed to better understand the balance of effector and regulatory $\mathrm{CD} 4{ }^{+} \mathrm{T}$-cells in mesothelioma. This would then also allow a linkage to mutational status and immune attack and targeting of such mutations, for example by vaccination.

A key $\mathrm{CD}^{+}{ }^{+} \mathrm{T}$-cell population that limits the function of effector and helper $\mathrm{T}$-cells are $\mathrm{FOXP} 3{ }^{+} \mathrm{CD} 4{ }^{+}$regulatory $\mathrm{T}$-cells. Consistent with the current understanding of their role, we observe that a high number of FOXP3 ${ }^{+}$T-cells was significantly associated with poorer survival in both morphological subgroups of mesothelioma. The effect of FOXP3 ${ }^{+}$T-reg likely has diseasespecific features. While in many solid tumour types a lower T-reg density is good for the patient (Shang et al, 2015), there are some exceptions, such as head and neck squamous cell carcinoma, where higher $\mathrm{FOXP}^{+}{ }^{+}$T-cell density is linked to better locoregional control (Badoual et al, 2006). It may simply be though that this apparent contradiction reflects a parallel influx of protective and suppressive immune cells, as in head and neck cancer where globally the number of immune cells is tightly linked to survival (Ward et al, 2014; Wood et al, 2016).

We evaluated, whether the sensitivity of quantification of the immune cell infiltrate could be improved by assessing the relative density of $\mathrm{CD}^{+}{ }^{+}$and $\mathrm{CD} 8{ }^{+}$T-cells. An easy way of expressing this is by calculating the ratio of $\mathrm{CD} 4^{+} / \mathrm{CD} 8^{+} \mathrm{T}$-cells, a measure that then becomes independent of the absolute abundance of the $\mathrm{T}$-cells in the tissue. A high $\mathrm{CD}^{+} / \mathrm{CD} 8^{+}$ratio has been previously evaluated in different types of cancer and was found to be associated with a good outcome in cervical squamous cell carcinoma and a poor outcome in colorectal cancer (Diederichsen et al, 2003; Shah et al, 2011). In our cohort, a CD4 ${ }^{+} / \mathrm{CD} 8{ }^{+}$ratio of greater than 1 was associated with better survival in the overall and epithelioid groups and this parameter warrants further analysis in other cancers to determine if it might be a useful prognostic marker for survival.

There is increasing recognition of the important role B-cells play in adaptive immune attack in the tumour microenvironment. Tumour-associated B-cells induce and regulate $\mathrm{T}$-cell immune responses through antigen presentation and $\mathrm{CD} 4{ }^{+} \mathrm{T}$-cell activation, contributing to the differentiation of $\mathrm{CD} 4{ }^{+}$T-cells and polarisation of Th1 and Th2 subsets (Lund and Randall, 2010; Baumgarth, 2011; Bao et al 2014). In our cohort, a high density of $\mathrm{CD}_{20}{ }^{+}$in the epithelioid group was associated with better survival. This remained significant on multivariate analysis and our data are consistent with Ujiie et al (2015). Similar to T-cells, there is variation in the prognostic effect of B-cell infiltration between tumour types. In colorectal cancer, an increased B-cell gene expression was significantly associated with survival (Bindea et al, 2013). Our own data in head and neck squamous cell carcinoma has demonstrated a high B-cell infiltrate to be associated with better outcome (Wood et al, 2016). It is unclear however what the B-cells might contribute immunologically, and whether are simply drawn to the tumour in response to cytokine stimulation.

Tumour-associated inflammation is recognised as a key hallmark of cancer (Grivennikov et al, 2010; Hanahan and Weinberg, 2011) and innate immune cells such as macrophages and neutrophils form an important and complex part of the tumour microenvironment. Tumour-associated macrophages can adopt a pro-tumourigenic role by promotion of angiogenesis and metastases and preventing T-cell attack on tumour cells and once the tumour is established, the macrophages are polarised to a protumour phenotype (Noy and Pollard, 2014). In our cohort, a low $\mathrm{CD} 8^{+}$count was significant in the epithelioid group, similar to findings by Ujiie et al (2015), but not in the non-epithelioid group. The association of a low infiltration of tumour-associated macrophages and survival is consistent with the theory of chronic asbestos-induced inflammation driving the development of mesothelioma in a process of 'frustrated phagocytosis' (Bograd et al, 2011).

Tumour-associated neutrophils similarly can adopt a protumourigenic role by involvement in angiogenesis and creating a pro-invasive and pro-metastatic environment and may also play an anti-tumourigenic role (Hanahan and Coussens, 2012). Consistent with this, in our cohort, a low number of NP57 cells were associated with better survival in the epithelioid group. To our knowledge, our group is the first to detect the prognostic value of a low neutrophil count on survival in epithelioid mesothelioma.

Beyond histological typing into epithelioid, sarcomatoid and mixed types, markers that predict response to treatment in 
mesothelioma are lacking. In other solid tumours such as breast, rectal and oesophageal cancer immunological features that predict for pathological complete response and survival following chemotherapy have been examined. In a breast cancer cohort, a pre-treatment high $\mathrm{CD}^{+} / \mathrm{CD}^{+}$ratio was an independent predictor of pathological complete response after neo-adjuvant chemotherapy and was associated with better prognosis (GarcíaMartínez et al, 2014) as was the presence of CD20 ${ }^{+}$B-cells (Brown et al, 2014). We therefore wondered if TIL density would link to treatment response in mesothelioma. We found that this was the case: in 61 patients who had received chemotherapy $\mathrm{CD} 4^{+} \mathrm{T}$-cell high cases identified patients who had a better outcome. This remained true also if only the 42 patients with epithelioid histology and who received chemotherapy were considered and suggest that $\mathrm{CD}^{+}{ }^{+} \mathrm{T}$-cells may also have a predictive value in mesothelioma.

In summary, our data demonstrate for the first time an association of survival with high $\mathrm{CD}^{+}$, low $\mathrm{FOXP}^{+}$, high $\mathrm{CD}_{20}{ }^{+}$, low $\mathrm{NP}_{57}^{+}$and low $\mathrm{CD}^{+} 8^{+}$counts in epithelioid mesothelioma, treated with palliative intent. Low $\mathrm{CD}^{+}{ }^{+}$and low $\mathrm{FOXP}^{+}{ }^{+}$T-cell densities emerge as prognostic in non-epithelioid mesothelioma. Given that epithelioid and non-epithelioid mesothelioma behave very differently clinically, the underpinning differences in potential immunological drivers of the different subtypes are intriguing and warrant further study and will benefit from mapping onto the emerging stratifiers using genomic analyses (Bueno et al, 2016). Emerging data suggesting that sarcomatoid mesothelioma may be more responsive to immunotherapy than the epithelioid subtype (Mansfield et al, 2014; Cedrés et al, 2015), but more work is needed to understand the reason behind this difference. Our data illustrate that the morphological differences, linked to outcome also find their reflection in the adaptive and innate immune events that are present in the cancer. Their functional understanding will open the door towards rational targeting of immune pathways to improve the outcomes of patients with this devastating disease.

\section{ACKNOWLEDGEMENTS}

This work was supported by the Mesothelioma Applied Research Foundation - The Lance S Ruble, Janelle Bedel and Ferraro Law Firm Grant; Cancer Research UK Programme Grant Reference C11512/A20256 and the NIHR Clinical Research Network.

${ }^{7}$ Joint senior authors.

\section{CONFLICT OF INTEREST}

The authors declare no conflict of interest.

\section{REFERENCES}

Alley EW, Lopez J, Santoro A, Morosky A, Saraf S, Piperdi B, van Brummelen E (2017) Clinical safety and activity of pembrolizumab in patients with malignant pleural mesothelioma (KEYNOTE-028): preliminary results from a non-randomised, open-label, phase $1 \mathrm{~b}$ trial. Lancet Onco 18(5): 623-630.

Al Shibli KI, Donnem T, Al-Saad S, Persson M, Bremnes RM, Busund LT (2008) Prognostic effect of epithelial and stromal lymphocyte infiltration in non-small-cell lung cancer. Clin Cancer Res 14(16): 5220-5227.

Anraku M, Cunningham KS, Yun Z, Tsao MS, Zhang L, Keshavjee S, Johnston MR, de Perrot M (2008) Impact of tumour-infiltrating T-cells on survival in patients with malignant pleural mesothelioma. J Thorac Cardiovasc Surg 135(4): 823-829.

Awad MM, Jones RE, Liu H, Lizotte PH, Ivanova EV, Kulkarni M, Herter-Sprie GS, Liao X, Santos AA, Bittinger MA, Keogh L, Koyama S, Almonte C, English JM, Barlow J, Richards WG, Barbie DA, Bass AJ, Rodig SJ, Hodi FS, Wucherpfennig KW, Jänne PA, Sholl LM,
Hammerman PS, Wong KK, Bueno R (2016) Cytotoxic T cells in PD-L1 positive malignant pleural mesotheliomas are counterbalanced by distinct immunosuppressive factors. Cancer Immunol Res 4(12): 1038-1048.

Badoual C, Hans S, Rodriguez J, Peyrard S, Klein C, Agueznay NEH, Mosseri V, Laccourreye O, Bruneval P, Fridman WH, Brasnu DF, Tartour E (2006) Prognostic value of tumour-infiltrating CD4 ${ }^{+}$T-cell subpopulations in head and neck cancers. Clin Cancer Res 12(2): 465-472.

Bao Y, Liu X, Han C, Xu S, Xie B, Zhang Q, Gu Y, Hou J, Qian L, Qian C, Han H, Cau X (2014) Identification of INF- $\gamma$-producing innate B cells. Cell Res 24(2): 161-176.

Baumgarth N (2011) The double life of a B-1 cell: self-reactivity selects for protective effector functions. Nat Rev Immunol 11(1): 34-46.

Bindea G, Mlecnik B, Tosolini M, Kirilovsky A, Waldner M, Obenauf AC, Angell H, Fredriksen T, Lafontaine L, Berger A, Bruneval P, Fridman WH, Becker C, Pagès F, Speicher MR, Trajanoski Z, Galon J (2013) Spatiotemporal dynamics of intratumoural immune cells reveal the immune landscape in human cancer. Immunity 39(4): 782-795.

Bograd AJ, Suzuki K, Vertes E, Colovos C, Morales EA, Sadelain M, Adusumilli PS (2011) Immune responses and immunotherapeutic interventions in malignant pleural mesothelioma. Cancer Immunol Immunother 60(11): 1509-1527.

Brown JR, Wimberly H, Lannin DR, Nixon C, Rimm DL, Bossuyt V (2014) Multiplexed quantitative analysis of $\mathrm{CD} 3, \mathrm{CD} 8$ and $\mathrm{CD} 20$ predicts response to neoadjuvant chemotherapy in breast cancer. Clin Cancer Res 20(23): 5995-6005.

Bueno R, Stawiski EW, Goldstein LD, Durinck S, De Rienzo A, Modrusan Z, Gnad F, Nguyen TT, Jaiswal BS, Chirieac LR, Sciaranghella D, Dao N, Gustafson CE, Munir KJ, Hackney JA, Chaudhuri A, Gupta R, Guillory J, Toy K, Ha C, Chen YJ, Stinson J, Chaudhuri S, Zhang N, Wu TD, Sugarbaker DJ, de Sauvage FJ, Richards WG, Seshagiri S (2016) Comprehensive genomic analysis of malignant pleural mesothelioma identifies recurrent mutations, gene fusions and splicing alterations. Nat Genet 48(4): 407-416.

Calabrò L, Morra A, Fonsatti E, Cutaia O, Amato G, Giannarelli D, Di Giacomo AM, Danielli R, Altomonte M, Mutti L, Maio M (2013) Tremelimumab for patients with chemotherapy-resistant advanced malignant mesothelioma: an open label, single-arm, phase 2 trial. Lancet Oncol 14(11): 1104-1111.

Cedrés S, Ponce-Aix S, Zugazagoitia J, Sansano I, Enguita A, Navarro-Mendivil A, Martinez-Marti A, Martinez P, Felip E (2015) Analysis of expression of programmed cell death ligand 1 (PD-L1) in malignant pleural mesothelioma (MPM). PLoS One 10(3): e0121071.

Comertpay S, Pastorino S, Tanji M, Mezzapelle R, Strianese O, Napolitano A, Baumann F, Weigel T, Friedberg J, Sugarbaker P, Krausz T, Wang E, Powers A, Gaudino G, Kanodia S, Pass HI, Parsons BL, Yang H, Carbone M (2014) Evaluation of clonal origin of malignant mesothelioma. J Transl Med 12: 301.

Diederichsen AC, Hjelmborg Jv, Christensen PB, Zeuthen J, Fenger C (2003) Prognostic value of the $\mathrm{CD} 4^{+} / \mathrm{CD} 8{ }^{+}$ratio of tumour infiltrating lymphocytes in colorectal cancer and HLA-DR expression on tumour cells. Cancer Immunol Immunother 52(7): 423-428.

Ding ZC, Blazar BR, Mellor AL, Munn DH, Zhou G (2010) Chemotherapy rescues tumour driven aberrant $\mathrm{CD} 4{ }^{+} \mathrm{T}$-cell differentiation and restores an activated polyfunctional helper phenotype. Blood 115(2): 2397-2406.

Dunn GP, Old LJ, Schreiber RD (2004) The three Es of cancer immunoediting. Annu Rev Immunol 22: 329-360.

Galon J, Costes A, Sanchez-Cabo F, Kirilovsky A, Mlecnik B, Lagorce-Pagès C, Tosolini M, Camus M, Berger A, Wind P, Zinzindohoué F, Bruneval P, Cugnenc PH, Trajanoski Z, Fridman WH, Pagès F (2006) Type, density, and location of immune cells within human colorectal tumours predict clinical outcome. Science 313(5795): 1960-1964.

García-Martínez E, Gil GL, Benito AC, González-Billalabeitia E, Conesa MA, García García T, García-Garre E, Vicente V, Ayala de la Peña F (2014) Tumour-infiltrating immune cell profiles and their change after neoadjuvant chemotherapy predict response and prognosis of breast cancer. Breast Cancer Res 16(6): 488.

Gentles AJ, Newman AM, Liu CL, Bratman SV, Feng W, Kim D, Nair VS, Xu Y, Khuong A, Hoang CD, Diehn M, West RB, Plevritis SK, Alizadeh AA (2015) The prognostic landscape of genes and infiltrating immune cells across human cancers. Nat Med 21(8): 938-945.

Gooden MJ, de Bock GH, Leffers N, Daemen T, Nijman HW (2011) The prognostic influence of tumour-infiltrating lymphocytes in cancer: a systematic review with meta-analysis. Br J Cancer 105(1): 93-103. 
Grivennikov SI, Greten FR, Karin M (2010) Immunity, inflammation and cancer. Cell 140(3): 883-899.

Hanahan D, Weinberg RA (2011) Hallmarks of cancer: the next generation. Cell 144(5): 646-674.

Hanahan D, Coussens LM (2012) Accessories to the crime: functions of cells recruited to the tumour microenvironment. Cancer Cell 21(3): 309-322.

Hassan R, Alley E, Kindler H, Antonia S, Jahan T, Thomas A, Honarmand S, Murphy AL, Grous JJ, Brockstedt DG (2015) Anti-mesothelin vaccine CRS-207 with or without low-dose cyclophosphamide plus chemotherapy as front line treatment for malignant pleural mesothelioma (MPM) J Immunother Cancer 3(Suppl 2): 161.

Jaurand MC, Renier A, Daubriac J (2009) Mesothelioma: Do asbestos and carbon nanotubes pose the same health risk? Part Fibre Toxicol 6: 16.

Kelly RJ, Sharon E, Pastan I, Hassan R (2012) Mesothelin-targeted agents in clinical trials and in pre-clinical development. Mol Cancer Ther 11(3): 517-525.

Lund FE, Randall TD (2010) Effector and regulatory B cells: modulators of CD4 + T cell immunity. Nat Rev Immunol 10(4): 236-247.

Mansfield AS, Roden AC, Peikert T, Sheinin YM, Harrington SM, Krco CJ, Dong H, Kwon ED (2014) B7-H1 expression in malignant pleural mesothelioma is associated with sarcomatoid histology and poor prognosis. J Thorac Oncol 9(7): 1036-1040.

Noy R, Pollard JW (2014) Tumour associated macrophages:from mechanism to therapy. Immunity 41(1): 49-61.

Rosenberg SA (2012) CAR T cell receptor immunotherapy targeting mesothelin for patients with metastatic cancer. Clinical Trials Identifier NCT01583686.

Scherpereel A, Astoul P, Baas P, Berghmans T, Clayson H, de Vuyst P, Dienemann H, Galateau-Salle F, Hennequin C, Hillerdal G, Le Péchoux C, Mutti L, Pairon JC, Stahel R, van Houtte P, van Meerbeeck J, Waller D, Weder W. European Respiratory Society/European Society of Thoracic Surgeons Task Force (2010) Guidelines of the European Respiratory Society and the European Society of Thoracic Surgeons for the management of malignant pleural mesothelioma. Eur Respir J 35(3): 479-495.

Schreiber RD, Old LJ, Smyth MJ (2011) Cancer immunoediting: integrating immunity's roles in cancer suppression and promotion. Science 331(6024): 1565-1570.

Shah W, Yan X, Jing L, Zhou Y, Chen H, Wang Y (2011) A reversed CD4/ CD8 ratio of tumour-infiltrating lymphocytes and a high percentage of $\mathrm{CD} 4(+)$ FOXP3 $(+)$ regulatory cells are significantly associated with clinical outcome in squamous cell carcinoma of the cervix. Cell Mol Immunol 8(1): 59-66.
Shang B, Liu Y, Jiang SJ, Liu Y (2015) Prognostic value of tumour infiltration $\mathrm{FOXP}^{+}$regulatory T-cells in cancers: a systematic review and metaanalysis. Sci Rep 5: 15179.

Testa JR, Cheung M, Pei J, Below JE, Tan Y, Sementino E, Cox NJ, Dogan AU, Pass HI, Trusa S, Hesdorffer M, Nasu M, Powers A, Rivera Z, Comertpay S, Tanji M, Gaudino G, Yang H, Carbone M (2011) Germline BAP1 mutations predispose to malignant mesothelioma. Nat Genet 43(10): 1022-1025.

Ujiie H, Kadota K, Nitadori JI, Aerts JG, Woo KM, Sima CS, Travis WD, Jones DR, Krug LM, Adusumilli PS (2015) The tumoural and stromal immune microenvironment in malignant pleural mesothelioma: A comprehensive analysis reveals prognostic immune markers. Oncoimmunology 4(6): e1009285.

Ward MJ, Thirdborough SM, Mellows T, Riley C, Harris S, Suchak K, Webb A, Hampton C, Patel NN, Randall CJ, Cox HJ, Jogai S, Primrose J, Piper K, Ottensmeier CH, King EV, Thomas GJ (2014) Tumour-infiltrating lymphocytes predict for outcome in HPV-positive oropharyngeal cancer. Br J Cancer. 110(2): 489-500.

Wood O, Woo J, Seumois G, Savelyeva N, McCann KJ, Singh D, Jones T, Peel L, Breen MS, Ward M, Garrido Martin E, Sanchez-Elsner T, Thomas G, Vijayanand P, Woelk CH, King E, Ottensmeier C. SPARC Consortium (2016) Gene expression analysis of TIL rich HPV-driven head and neck tumours reveals a distinct B-cell signature when compared to HPV independent tumours. Oncotarget 7(35): 56781-56797.

Yamada N, Oizumi S, Kikuchi E, Shinagawa N, Konishi-Sakakibara J, Ishimine A, Aoe K, Gemba K, Kishimoto T, Torigoe T, Nishimura M (2010) CD8 + tumour-infiltrating lymphocytes predict favorable prognosis in malignant pleuralmesothelioma after resection. Cancer Immunol Immunother 59(10): 1543-1549.

Zauderer M (2010) Randomized study of adjuvant WT1 analog peptide vaccine in patients with malignant pleural mesothelioma (MPM) after completion of combined modality therapy. Clinical Trials identifier NCT01265433.

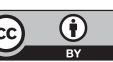

This work is licensed under the Creative Commons Attribution 4.0 International License. To view a copy of this license, visit http://creativecommons.org/licenses/by/4.0/

(C) The Author(s) named above 2017

Supplementary Information accompanies this paper on British Journal of Cancer website (http://www.nature.com/bjc) 